\title{
Pricing Games in Multihop Wireless Networks under Interference Constraints
}

\author{
Anil Kumar Chorppath, Edmund M. Yeh and Holger Boche
}

\begin{abstract}
In this paper, we consider a multihop wireless network, where Femto Base Stations (FBSs) act as relay nodes, and are incentivized to carry traffic from a Macro Base Station (MBS) to Macro Users (MUs). We first examine the the global problem of jointly optimal allocation of traffic flow and transmission power in the multihop wireless network. We then examine a game in which selfish and strategic relays submit charging functions to the source and choose transmission powers over a MAC channel from the relays to the user. Relay charging functions are considered which yield efficient allocation at the Nash Equilibrium (NE) of the game. We observe that for efficiency, relays should be taxed for the interference it creates to other relays. We also observe that inefficient equilibria occur when the charging function is a function only of the traffic flow rate through the relay. Numerical studies demonstrate the variation of inefficiency with network structure.
\end{abstract}

\section{INTRODUCTION}

Due to their ability to bring about massive spatial reuse of frequency, small cell base stations such as Femto Base Stations (FBS) or Pico Base Stations (PBS) are increasingly important for improving network capacity. At the same time, FBSs also give better data rate to end users due to short transmission range and fewer users per cell. FBSs are normally deployed in indoor home or office environments owned or rented by second parties other than the service provider, and are normally underutilized. One way to better utilize the capacities of FBSs is to employ the FBSs as relays [1]. In this scenario, FBSs carry traffic from the Macro Base Station (MBS) to Macro Users (MUs), in addition to serving Femto Users (FUs). The relaying generates revenue for the owner of the FBSs. Moreover, the relaying extends the coverage of FBSs to outdoor environments [2], thereby reducing the burden on the MBS.

Incentives play a large role in reconciling the objectives of selfish and strategic nodes to the global objectives of the network, which may include the efficient allocation of network resources [3]. In [4], a pricing game is considered within a multi-hop relay network where link cost functions depend only on the traffic flow rate. Each relay submits to the Source a charging function and a demand for a traffic share. The paper [5] investigates pricing games with both complete and incomplete information within multihop wireless networks,

The work by Anil Kumar Chorppath is supported by the COIN project by German National Science Foundation (DFG) BO 1734/24-1. The work by Edmund M. Yeh and Holger Boche is supported by the Alexander von Humboldt Foundation. Anil Kumar Chorppath and Holger Boche are with Technical University of Munich, 80333 Munich, Germany. Edmund M. Yeh is with the Department of Electrical and Computer Engineering, Northeastern University, Boston, MA 02461, USA. without taking into account the interference coupling between relays. For the complete information case, it is shown that all NE's are efficient and that there exists an efficient NE where each relay uses a charging function which depends linearly on the traffic flow rate. In [6], cooperative relaying is considered where the relays are incentivized to forward packets within a Stackelberg game framework. A bargaining game with utility requirements is considered for the same scenario in [7]. In another relevant work, the authors of [8] proposed, within a wireless ad hoc network setting, an asynchronous pricing algorithm in which each user cooperatively announces a price to which all users respond by adjusting their transmission powers. The price announced by each user reflects its sensitivity to the interference created by other users.

We consider a wireless network scenario where an MBS (Source) uses multiple relay nodes owned by some second parties to give better service to the MUs. A specific instance of the problem is given in Figure 1, with an MBS, two relays and an MU. The MU at the cell edge is better served using the two relays, an FBS and a PBS, which are rewarded by the MBS for forwarding the traffic to the MU. In this wireless

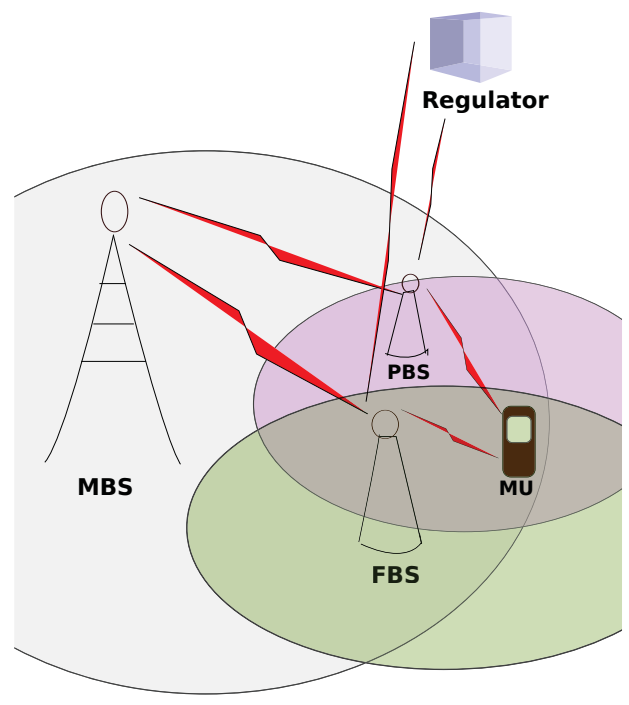

Fig. 1. A Pico Base Station (PBS) and a Femto Base Station (FBS) acting as relays for the Macro Base Station (MBS) to give service to user at the cell edge. 
network setting, selfish and strategic relays engage in a game where each relay strategize on the charging function they submit to the source, as well as the transmission power to the end user. The transmissions on different wireless links in this multihop network interfere with each other, with the link capacities dependent on the power allocation on all links. Each relay designs its best response based on its cost, according to the traffic flow rate and transmission power allocated by the source, as well as the charging functions and power selections of the other relays. We examine the Nash Equilibria (NE) which result from this game, which may not yield an efficient resource allocation from a social welfare standpoint. We use the Price of Anarchy (PoA) [9] as the metric to characterize the inefficiency of the resource allocation at the NE, as compared with the socially optimal allocation.

Within the game setting discussed above, we introduce the concept of interference tax which the relays should pay to an external regulator as compensation for the interference they create to other relays. We prove that inefficient NE occur when the price is a function only of the traffic flow rate through the relays. On the other hand, we prove that there exists at least one NE which is efficient when the charging function depends on both the traffic flow rate and the transmission powers from the relays to the destination. Our result highlights the importance of interference coupling among the relays in determining network resource allocation in a selfish and strategic context.

\section{MODEL}

We consider a network in which a Source transmits to a destination through $\{1, \cdots, i, \cdots N\}$ parallel relays. The model is depicted in Figure 2. The cost on link $(s, i)$ from Source $s$ to relay $i$, denoted by $J_{s i}$, is the sum of two components: the congestion cost $D_{s i}$ and the power cost $\alpha_{s} P_{s i}$. The congestion cost $D_{s i}$ is a function of the capacity $C_{s i}$ of link $(s, i)$, and the traffic flow rate $F_{i}$ on link $(s, i)$. The power cost $\alpha_{s} P_{s i}$ consists of the balancing parameter $\alpha_{s} \in \Re^{+}$ and the transmission power $P_{s i}$ from Source $s$ to relay $i$.

Unlike the case for wireline networks, the capacity of a wireless link is not fixed, but rather depends on the channel conditions and the transmission powers. The joint power and rate allocation for the links from the Source to the relays is carried out by the Source.

For the case of spread spectrum CDMA, the Shannon capacity $C_{s i}$ is given by

$$
C_{s i}\left(\mathbf{P}_{s}\right)=\frac{R_{s}}{2} \log \left(1+\frac{K h_{s i} P_{s i}}{\sum_{j \neq i} h_{s j} P_{s j}+\sigma}\right),
$$

where $\mathbf{P}_{s} \equiv\left(P_{s 1}, \ldots, P_{s N}\right), h_{s i}$ is the channel gain from Source $s$ to relay $i, R_{s}$ is the symbol rate, $K$ is the code gain, and $\sigma$ is the noise power at the receiver of $i$. Since the code gain $K$ is typically high, we assume that the operation is in the high SINR regime, where the capacity can be approximated

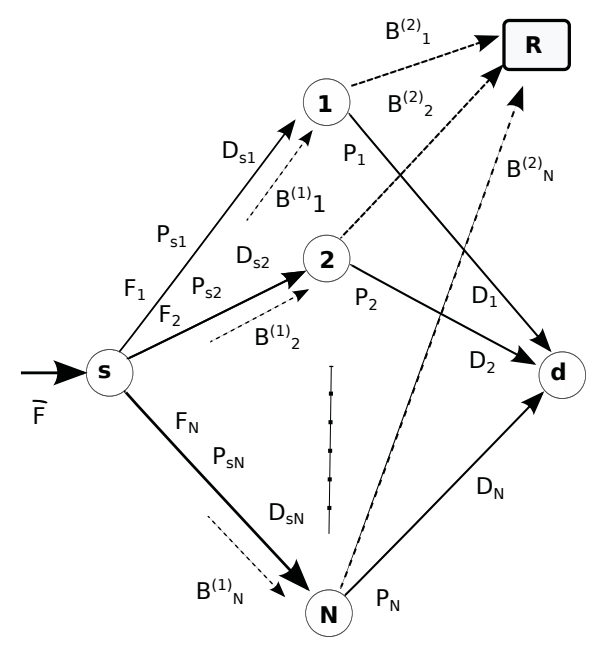

Fig. 2. Multihop wireless network model with $N$ relays. $F_{i}$ is the flow rate on link $i ; P_{s i}$ is the transmission power from Source $s$ to relay $i ; P_{i}$ is the transmission power from relay $i$ to destination $d ; D_{s i}$ is the congestion cost from $s$ to $i ; D_{i}$ is the congestion cost from $i$ to $d ; B_{i}^{(1)}$ is the payment paid by $s$ to relay $i$; Regulator $R$ receives the payments $B_{i}^{(2)}$ from the relays.

by

$$
C_{s i}\left(\mathbf{P}_{s}\right) \approx \frac{R_{s}}{2} \log \left(\frac{K h_{s i} P_{s i}}{\sum_{j \neq i} h_{s j} P_{s j}+\sigma}\right) .
$$

This approximation, along with the following assumption, facilitates the convexification of the optimization problems arising later in this paper.

Assumption II.1. The congestion cost $D_{s i}\left(C_{s i}, F_{i}\right)$ is a twice differentiable, convex and increasing function of $F_{i}$, and a twice differentiable, convex and decreasing function of $C_{s i}$.

Let the payment paid by the Source to the relay $i$ be $B_{i}$. As discussed below, $B_{i}$ can be a function of the flow rate $F_{i}$ carried by relay $i$, as well as other variables. Thus, the Source incurs the total cost

$$
J_{s}\left(\mathbf{P}_{s}, \mathbf{F}\right)=\sum_{i=1}^{N}\left[D_{s i}\left(C_{s i}\left(\mathbf{P}_{s}\right), F_{i}\right)+\alpha_{s} P_{s i}+B_{i}\right] .
$$

We assume that the source-to-relay communication channel is orthogonal to the relay-to-destination communication channel. Let $\mathbf{P} \equiv\left(P_{1}, \ldots, P_{N}\right)$, where $P_{i}$ is the transmission power from relay $i$ to the destination. The capacity of the wireless link from relay $i$ to the destination is approximated by

$$
C_{i}(\mathbf{P}) \approx \frac{R_{s}}{2} \log \left(\frac{K h_{i} P_{i}}{\sum_{j \neq i} h_{j} P_{j}+\sigma}\right),
$$

where $h_{i}$ is the channel gain from relay $i$ to the destination, and $\sigma$ is the noise power at the destination receiver. Therefore, each 
relay $i$ has a total cost of transmission $J_{i}$ to the destination, given by

$$
J_{i}\left(\mathbf{P}, F_{i}\right)=D_{i}\left(C_{i}(\mathbf{P}), F_{i}\right)+\alpha_{i} P_{i}-B_{i} .
$$

where $D_{i}$ satisfies Assumption II.1 and $\alpha_{i}$ is a balancing parameter. Note that the total cost over parallel path $i$ is given by

$$
D_{s i}\left(C_{s i}\left(\mathbf{P}_{s}\right), F_{i}\right)+D_{i}\left(C_{i}(\mathbf{P}), F_{i}\right)+\alpha_{s} P_{s i}+\alpha_{i} P_{i} .
$$

The power allocation from the Source to the relays is accomplished centrally at the Source, whereas the transmission powers from the relays to the destination are selected in a distributed manner by the relays. Each relay $i$ competes with all other relays by selecting the payment function $B_{i}$ and transmission power $P_{i}$, in order to maximize its net profit, which is equal to the revenue generated by forwarding a share of the traffic from the Source to the destination, minus the power expense required for forwarding the traffic. This is the basis of the game among the relays.

All relays should gain positive net utility by participating in the game, a condition usually known as the Individual Rationality (IR) property [10]. The IR constraint in the relay problem is given by: $J_{i}\left(P_{i}\right) \leq 0$ for all $i=1, \ldots, N$. The IR constraint is satisfied through the following process. First, the relays submit charging functions. Then, the source decides on the optimal flows in response to these payment functions, without taking into account the IR constraint on each relay. Given this, some relays may find that they cannot find a positive finite power which will ensure negative cost, for the flow given by the source and power chosen by other relays. This might happen, for instance, if the channel gains from those relays to the destination are very low compared to others. In this case, these relays will opt out of the game and will report this to the source. Subsequently, the source will reallocate its flows excluding the non-participating relays. This process continues until a stable subset of relays satisfying the IR constraint emerges.

\section{A. Joint global optimization of power and flow}

The aggregate network cost is the sum of the costs on all parallel paths. Therefore, the global optimization for obtaining the socially optimal (efficient) operating point is given by

$$
\begin{array}{r}
\min _{\mathbf{P}_{s}, \mathbf{P}, \mathbf{F}} \sum_{i=1}^{N}\left[D_{s i}\left(C_{s i}\left(\mathbf{P}_{s}\right), F_{i}\right)+\alpha_{s} P_{s i}+D_{i}\left(C_{i}(\mathbf{P}), F_{i}\right)+\alpha_{i} P_{i}\right] \\
\text { s.t. } \sum_{i} F_{i}=\bar{F}, F_{i} \geq 0, P_{i} \geq 0, P_{s i} \geq 0, \text { for all } i
\end{array}
$$

This problem can be shown to be jointly convex in the $\log$ power variables $\mathbf{S}_{s}=\log \left(\mathbf{P}_{s}\right), \mathbf{S}=\log (\mathbf{P})$, and the flow vector $\mathbf{F}$ using the high SINR assumption and Assumption II.1. After the log transformation, the global optimization becomes

$$
\begin{array}{r}
\min _{\mathbf{S}_{s}, \mathbf{S}, \mathbf{F}} \sum_{i=1}^{N}\left[D_{s i}\left(C_{s i}\left(\mathbf{S}_{s}\right), F_{i}\right)+\alpha_{s} e^{S_{s i}}+D_{i}\left(C_{i}(\mathbf{S}), F_{i}\right)+\alpha_{i} e^{S_{i}}\right] \\
\text { s.t. } \sum_{i} F_{i}=\bar{F}, F_{i} \geq 0 \forall i .
\end{array}
$$

The above problem leads to the following KKT conditions for global optimality. For each $i=1, \ldots, N$,

$$
\begin{gathered}
\frac{\partial D_{s i}}{\partial F_{i}}+\left.\frac{\partial D_{i}}{\partial F_{i}}\right|_{\mathbf{F}=\mathbf{F}^{g}}=d^{g} \text { if } F_{i}^{g}>0, \\
\frac{\partial D_{s i}}{\partial F_{i}}+\left.\frac{\partial D_{i}}{\partial F_{i}}\right|_{\mathbf{F}=\mathbf{F}^{g}}>d^{g} \text { if } F_{i}^{g}=0, \\
\frac{\partial D_{s i}}{\partial S_{s i}}+\sum_{j \neq i} \frac{\partial D_{s j}}{\partial S_{s i}}+\left.\alpha_{s} e^{S_{s i}}\right|_{\mathbf{S}_{s}=\mathbf{S}_{s}^{g}}=0, \\
\frac{\partial D_{i}}{\partial S_{i}}+\sum_{j \neq i} \frac{\partial D_{j}}{\partial S_{i}}+\left.\alpha_{i} e^{S_{i}}\right|_{\mathbf{S}=\mathbf{S}^{g}}=0,
\end{gathered}
$$

where $\mathbf{P}_{s}^{g}, \mathbf{P}^{g}, \mathbf{F}^{g}$ are the globally optimal source transmission power vector, relay transmission power vector, and flow rate vector, respectively, and $d^{g}$ is a constant which corresponds to the optimal operating point.

\section{PRICING GAME}

In this section, we analyze the pricing game in which the relays compete with each other for the traffic allocation from the Source by strategizing on the charging function and the power of transmission to the destination. Each relay sends a charging function $B_{i}$ to the Source, which performs the flow allocation and power allocation to the relays according to the charging function.

As a first step, as in [5], we assume that the Source is charged as a function of the flow rates it sends through the relays, i.e., the charging functions are given by $B_{i}\left(F_{i}\right)$. The Source optimization problem is to minimize the cost given by (2), i.e.,

$$
\begin{array}{r}
\min _{\mathbf{P}_{s}, \mathbf{F}} \sum_{i}\left(D_{s i}\left(C_{s i}\left(\mathbf{P}_{s}\right), F_{i}\right)+\alpha_{s} P_{s i}+B_{i}\left(F_{i}\right)\right), \\
\quad \text { s.t. } \sum_{i} F_{i}=\bar{F}, F_{i} \geq 0 \forall i \text { and } P_{s i} \geq 0 \forall i
\end{array}
$$

The Source decides on the flow and power vectors on each link to the relays by solving the above problem for every set of charging functions given by the relays. This problem can be shown to be jointly convex in high SINR region, in the log power variables $\mathbf{S}_{s}=\log \left(\mathbf{P}_{s}\right)$ and the flow vectors $\mathbf{F}$ if the payment is a convex function of the flow. The Source KKT conditions are:

$$
\begin{gathered}
\frac{\partial D_{s i}}{\partial F_{i}}+\left.\frac{\partial B_{i}}{\partial F_{i}}\right|_{\mathbf{F}=\mathbf{F}^{*}}=d^{*} \text { if } F_{i}^{*}>0, \\
\frac{\partial D_{s i}}{\partial F_{i}}+\left.\frac{\partial B_{i}}{\partial F_{i}}\right|_{\mathbf{F}=\mathbf{F}^{*}}>d^{*} \text { if } F_{i}^{*}=0, \forall i \\
\frac{\partial D_{s i}}{\partial S_{s i}}+\sum_{j \neq i} \frac{\partial D_{s j}}{\partial S_{s i}}+\left.\alpha_{s} e^{S_{s i}}\right|_{\mathbf{S}_{s}=\mathbf{S}_{s}^{*}}=0, \forall i
\end{gathered}
$$


where $\mathbf{P}_{s}^{*}=e^{\mathbf{S}_{s}^{*}}$ and $\mathbf{F}^{*}$ give the solution of the Source optimization and $d^{*}$ corresponds to the Source optimal operating point.

In the associated game, relays strategize using the charging functions, B, and the transmission power to the end user, $\mathbf{P}$. The relays are implicitly competing over the transmission powers due to the interference they cause to each other. The relays also compete for the traffic flow using the payment they charge from the Source. Due to the coupling in the cost term $D_{i}\left(C_{i}(\mathbf{P}), \mathbf{F}_{i}\right)$, we can think of the relays as deciding on the charging function and the transmission powers at the same time. The strategy space of each relay is infinite dimensional in general.

The NE of this game can be obtained from the intersection of the best responses of all relays, given by

$$
\left(\mathbf{S}_{i}^{*}, \mathbf{B}_{i}^{*}\right) \in \arg \min _{S_{i}, B_{i}} J_{i}\left(S_{i}, B_{i}, \mathbf{S}_{-i}, \mathbf{B}_{-i}\right), \forall i .
$$

Definition III.1. A NE is efficient if the flow rate allocation and power allocation at the NE solves the global optimization.

In the multihop wireless problem we consider, an NE is efficient when $\mathbf{S}^{*}=\mathbf{S}^{g}, \mathbf{F}^{*}=\mathbf{F}^{g}, \mathbf{S}_{s}^{*}=\mathbf{S}_{s}^{g}$, where $\left(\mathbf{S}_{s}^{*}, \mathbf{F}^{*}\right)$ is the solution to the source optimization problem when the relays present the NE charging functions $\mathbf{B}^{*}$ from (11) to the Source. The following Proposition shows that this situation cannot obtain when the charging function depends only on the traffic flow rate.

Proposition III.2. A Nash Equilibrium of the pricing game cannot be efficient when the relay charging functions depend only on the traffic flow rate carried by the relays.

Proof: Suppose that there exists an efficient Nash equilibrium $\mathbf{S}^{*}, \mathbf{B}^{*}$ with the charging function $B_{i}\left(F_{i}\right)$. The Source solves the following optimization problem to find the Source optimal power and flow

$$
\begin{array}{r}
\left(\mathbf{S}_{s}^{*}, \mathbf{F}^{*}\right)=\arg \min _{\mathbf{S}_{s}, \mathbf{F}} \sum_{i}\left(D_{s i}\left(C_{s i}\left(\mathbf{S}_{s}\right), F_{i}\right)+\alpha_{s} e^{S_{s i}}+B_{i}^{*}\left(F_{i}\right)\right) \\
\text { s.t. } \sum_{i} F_{i}=\bar{F}, F_{i} \geq 0 \forall i
\end{array}
$$

This results in the following KKT conditions.

$$
\begin{aligned}
\frac{\partial D_{s i}}{\partial F_{i}}+\left.\frac{\partial B_{i}^{*}}{\partial F_{i}}\right|_{\mathbf{F}=\mathbf{F}^{*}}=d^{*} \text { if } F_{i}^{*} & >0, \\
\frac{\partial D_{s i}}{\partial F_{i}}+\left.\frac{\partial B_{i}^{*}}{\partial F_{i}}\right|_{\mathbf{F}=\mathbf{F}^{*}}>d^{*} \text { if } F_{i}^{*} & =0, \forall i \\
\frac{\partial D_{s i}}{\partial S_{s i}}+\sum_{j \neq i} \frac{\partial D_{s j}}{\partial S_{s i}}+\left.\alpha_{s} e^{S_{s i}}\right|_{\mathbf{S}_{s}=\mathbf{S}_{s}^{*}} & =0, \forall i
\end{aligned}
$$

Once the relays receive the allocation $\mathbf{S}_{s}^{*}, \mathbf{F}^{*}$ from the Source, they minimizes the relay cost in equation (3) to find the best response.

$$
\mathbf{S}_{i}^{*}=\arg \min _{\mathbf{S}_{i}} D_{i}\left(C_{i}\left(S_{i}, \mathbf{S}_{-i}^{*}\right), F_{i}^{*}\right)+\alpha_{i} e^{S_{i}}-B_{i}^{*}\left(F_{i}^{*}\right) \forall i .
$$

The KKT conditions for the relay optimization w.r.t. $\mathbf{S}$ which give the NE $\mathbf{S}^{*}$ are:

$$
\frac{\partial D_{i}\left(C_{i}\left(S_{i}, \mathbf{S}_{-i}^{*}\right), F_{i}^{*}\right)}{\partial S_{i}}+\left.\alpha_{i} e^{S_{i}}\right|_{\mathbf{S}=\mathbf{S}^{*}}=0, \forall i
$$

From the Definition III.1, for the NE point to be efficient, the solution of (5) (6) and (7) and the joint solution of (12),(13), and (15) should be the same. We observe that it is not enough to set

$$
\left.\frac{\partial B_{i}^{*}}{\partial F_{i}}\right|_{\mathbf{F}=\mathbf{F}^{*}}=\left.\frac{\partial D_{i}}{\partial F_{i}}\right|_{\mathbf{F}=\mathbf{F}^{g}}, \forall i
$$

as in [5]. Indeed, we can see from the KKT conditions w.r.t. $\mathbf{S}$ in (7) and (15), that the resulting solutions are different, as the second (interference) term in (7) is not accounted for in (15). Therefore, the KKT conditions of the NE solution and globally optimal solution are not aligned. In general, $\mathbf{S}^{*} \neq$ $\mathbf{S}^{g}, \mathbf{F}^{*} \neq \mathbf{F}^{g}, \mathbf{S}_{s}^{*} \neq \mathbf{S}_{s}^{g}$ and the NE cannot be efficient.

\section{A. Pricing Function for Efficient NE}

We now consider an alternative approach. Assume that the charging function depends on both the traffic flow rate forwarded by the relay and the power the relay spends for the relaying, i.e. $B_{i}\left(F_{i}, P_{i}\right)$. Specifically, we split the charging function as follows: $B_{i}\left(F_{i}, P_{i}\right)=B_{i}^{(1)}\left(F_{i}\right)-B_{i}^{(2)}\left(P_{i}\right)$. The payment $B_{i}^{(1)}\left(F_{i}\right)$ is paid by the Source to the relay, while the payment $B_{i}^{(2)}\left(P_{i}\right)$ is paid by the relay to a centralized controller.

Theorem III.3. There exists an efficient $N E$ in the pricing game where the charging function is given by $B_{i}\left(F_{i}, S_{i}\right)=$ $B_{i}^{(1)}\left(F_{i}\right)-B_{i}^{(2)}\left(S_{i}\right)$, satisfying

$$
\left.\frac{\partial B_{i}^{(1) *}}{\partial F_{i}}\right|_{\boldsymbol{F}=\boldsymbol{F}^{*}}=\left.\frac{\partial D_{i}\left(\boldsymbol{P}, F_{i}\right)}{\partial F_{i}}\right|_{\boldsymbol{F}=\boldsymbol{F}^{g}}, \forall i
$$

and

$$
\left.\frac{\partial B_{i}^{(2) *}}{\partial S_{i}}\right|_{\boldsymbol{S}=\boldsymbol{S}^{*}}=\left.\sum_{j \neq i} \frac{\partial D_{j}\left(\boldsymbol{P}, F_{j}\right)}{\partial S_{i}}\right|_{\boldsymbol{S}=\boldsymbol{S}^{g}}, \forall i .
$$

where $\left(\boldsymbol{S}^{*}, B^{(1) *}, B^{(2) *}, \boldsymbol{F}^{*}\right)$ is the $N E$ and $\left(\boldsymbol{S}^{g}, \boldsymbol{F}^{g}\right)$ is the global optimum.

Proof: A standard result in game theory (Theorem 4.4, p.176, in [11]) for convex cost functions which satisfy Assumptions II.1, proves that the game we consider admits a NE.

Given the payment charge $B^{(1) *}\left(F_{i}\right)$ from the relays, the Source cost becomes

$$
J_{s}=\sum_{i}\left(D_{s i}\left(C_{s i}\left(\mathbf{S}_{s}\right), F_{i}\right)+\alpha_{s} e^{S_{s i}}+B_{i}^{(1) *}\left(F_{i}\right)\right) .
$$


The KKT conditions for Source optimization are

$$
\begin{aligned}
& \frac{\partial D_{s i}}{\partial F_{i}}+\left.\frac{\partial B_{i}^{(1) *}}{\partial F_{i}}\right|_{\mathbf{F}=\mathbf{F}^{*}}=d^{*} \text { if } F_{i}^{*}>0, \\
& \frac{\partial D_{s i}}{\partial F_{i}}+\left.\frac{\partial B_{i}^{(1) *}}{\partial F_{i}}\right|_{\mathbf{F}=\mathbf{F}^{*}}>d^{*} \text { if } F_{i}^{*}=0, \forall i \\
& \frac{\partial D_{s i}}{\partial S_{s i}}+\sum_{j \neq i} \frac{\partial D_{s j}}{\partial S_{s i}}+\left.\alpha_{s} e^{S_{s i}}\right|_{\mathbf{S}_{s}=\mathbf{S}_{s}^{*}}=0, \forall i
\end{aligned}
$$

where $\mathbf{F}^{*}, \mathbf{S}_{s}^{*}$ is the source-optimal solution. With this allocation from the Source, the relays carry out their optimizations

$$
\begin{aligned}
S_{i}^{*}=\arg \min _{S_{i}} & D_{i}\left(C_{i}, F_{i}\right)+\alpha_{i} e^{S_{s i}} \\
- & B_{i}^{(1) *}\left(F_{i}\right)+B_{i}^{(2) *}\left(S_{i}\right), \forall i
\end{aligned}
$$

The KKT condition for relay optimization is,

$$
\frac{\partial D_{i}}{\partial S_{i}}+\alpha_{i} e^{S_{i}}+\left.\frac{\partial B_{i}^{(2) *}}{\partial S_{i}}\right|_{S_{i}=S_{i}^{*}}=0, \forall i
$$

In order to obtain an efficient NE, from (5) and (19), we obtain the condition in (16). From (7) and (22), we obtain the condition in (17).

The relays receive payments from the Source for the traffic flow they carry, and are charged for the interference they create to other relays. We introduce an outside regulator which collects the interference tax payments from the relays. As an instance of the pricing given in (17), we propose logarithmic pricing on power for the interference tax. The logarithmic pricing is also similar to the universal pricing proposed in [12] for a different setting other than multihop wireless networks and without flow variables. The authors in [12] show that logarithmic pricing in power is universal pricing for systems with certain class of interference coupled utility functions. We set

$$
B_{i}\left(F_{i}, P_{i}\right)=a_{i} F_{i}-b_{i} \log \left(P_{i}\right) .
$$

According to (16) and (17), the coefficients are given by,

$$
a_{i}=\left.\frac{\partial D_{i}}{\partial F_{i}}\right|_{\mathbf{F}=\mathbf{F}^{g}}, \forall i
$$

and

$$
b_{i}=\left.\sum_{j \neq i} \frac{\partial D_{j}}{\partial S_{i}}\right|_{\mathbf{S}=\mathbf{S}^{g}}, \forall i .
$$

Note that due to the high SINR assumption, $P_{i}>1$ for any relay $i$, and the logarithmic power price is positive.

\section{B. PoA When Price is a Function Only of the Flow}

We now examine the inefficiency of the NE when the charging function is a function only of the traffic flow rate through the link.

Definition III.4. The Price of Anarchy (PoA) for an NE denoted by $\left(\boldsymbol{P}_{s}^{*}, \boldsymbol{P}^{*}, \boldsymbol{B}^{*}, \boldsymbol{F}^{*}\right)$ is defined as

$$
P o A=\frac{J_{g}\left(\boldsymbol{P}_{s}^{*}, \boldsymbol{P}^{*}, \boldsymbol{F}^{*}\right)}{J_{g}\left(\boldsymbol{P}_{s}^{g}, \boldsymbol{P}^{g}, \boldsymbol{F}^{g}\right)}
$$

where $J_{g}$ denotes the global cost and $\left(\boldsymbol{P}_{s}^{g}, \boldsymbol{P}^{g}, \boldsymbol{F}^{g}\right)$ is the globally optimal solution.

For interference coupled systems, we have observed that efficiency loss results when the charging function is a function only of the traffic flow rate, as in the case of [5]. We now consider a two-relay example with charging functions which depend linearly on the traffic flow rate, i.e. $B_{i}\left(F_{i}\right)=a_{i} F_{i}$, where $a_{i}$ is given by (23). In this case, $P_{s 1}^{*}, P_{s 2}^{*}, F_{1}^{*}$ are obtained from (12) and (13), while $P_{1}^{*}, P_{2}^{*}$ result from the following relay KKT conditions:

$$
\begin{aligned}
& \frac{\partial D_{1}\left(\mathbf{S}, F_{1}^{*}\right)}{\partial S_{1}}+\left.\alpha_{1} S_{1}\right|_{S_{1}=S_{1}^{*}}=0 \\
& \frac{\partial D_{2}\left(\mathbf{S}, F_{1}^{*}\right)}{\partial S_{2}}+\left.\alpha_{2} S_{2}\right|_{S_{2}=S_{2}^{*}}=0 .
\end{aligned}
$$

Since it is not easy to solve these equations analytically, we solve the equations numerically. The resulting $P_{o} A$ is plotted in the next section.

\section{Numerical Simulation}

We numerically simulate the range of values of $\operatorname{Po} A$ for the two-relay example described in previous section. The $\bar{F}$ parameter is fixed at 6 . First, the values $a_{1}$ and $a_{2}$ are selected as the marginal cost w.r.t. to the flows at the globally optimal point. Then, the source optimization problem is solved using $a_{1}$ and $a_{2}$ to find the flow and source power vector at the NE point. At the values of $F_{1}$ and $F_{2}$ given by the source optimum, the NE power vectors are found by the relays individually. The power vectors are different from the global point and give rise to PoA values as shown in Figure 3. The channel parameters $h_{s 2}=h_{2}$ are set to 5. The parameters $h_{1}$ and $h_{s 1}$ are varied from 5 to 180 . We stop at $h_{1}=h_{s 1}=180$ since it is observed that after 180 the link from Relay 2 to the destination cannot have positive capacity, and due to the IR constraint, Relay 2 opts out from the game. The optimal flow allocation at the NE is also shown in Figure 3.

As the link to relay 1 has higher channel gain compared to the link to relay 2 , there is more flow allocated to relay 1. We can observe that the PoA increases as the channel gains become dissimilar. In the asymmetric case, when the Source finds the optimal flow, it does not take into account the asymmetry of the channel gains from relays to the destination, since the price is only a function of the flow. On the other hand, the global allocation is affected by the asymmetry of the channel gains from relays to the destination. For this reason, there is a higher $P o A$ when the channel gains from the relays to the destination are asymmetric. This is numerically shown in Figure 3.

Finally, we show the variation of $\operatorname{PoA}$ with the number of relays $N$ in Figure 4 . The parameters are selected as above with $h_{s 1}=h_{1}=20$ and $h_{s 2}=h_{2}=5$, with the number of relays varying up to 20 . We can see that the $P o A$ increases as the number of relays increases. 


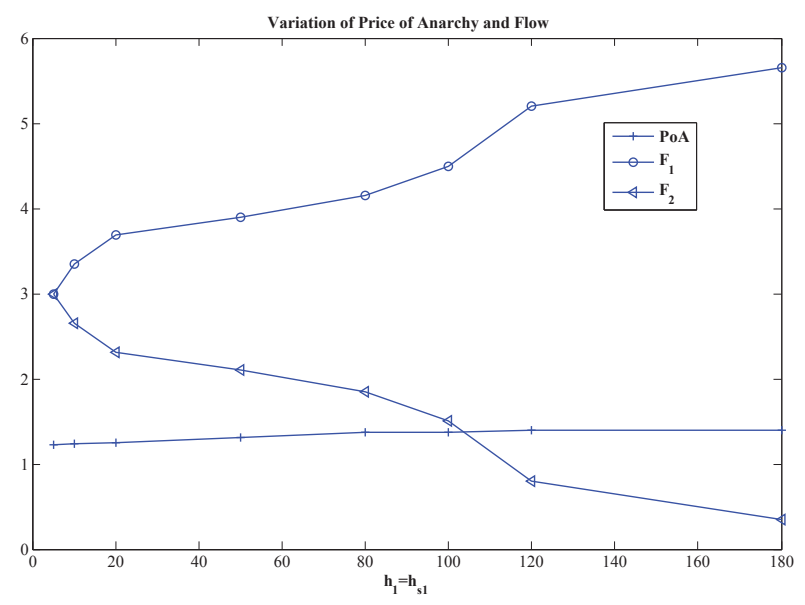

Fig. 3. The $P o A$ and path flows as functions of the channel gain in path 1 .

\section{CONCLusion}

A multihop wireless network is considered in which FBS or PBS relays receive payment from the MBS in return for traffic they forward to the MUs. We first examined the global problem of jointly optimizing power and rate allocation in the multihop wireless network. We then analyzed a pricing game among the relays and studied the efficiency of the NE for different choices of charging functions. Unlike the wireline case, the charging function for efficient allocation is seen to be a function of the transmission power as well as the traffic flow. We proposed a power price in the form of an interference tax which should be collected by an external regulator from the relays. The values of $P o A$ when the charging function is a function only of the flow are numerically obtained. We observed, using numerical analysis, that the $P o A$ increases with a higher number of relays and asymmetry in the network.

\section{REFERENCES}

[1] T. Elkourdi and O. Simeone, "Femtocell as a relay: An outage analysis," Wireless Communications, IEEE Transactions on, vol. 10, no. 12, pp. 4204-4213, 2011.

[2] C. Gueguen, A. Rachedi, and M. Guizani, "Incentive scheduler algorithm for cooperation and coverage extension in wireless networks," Vehicular Technology, IEEE Transactions on, vol. 62, no. 2, pp. 797-808, Feb 2013.

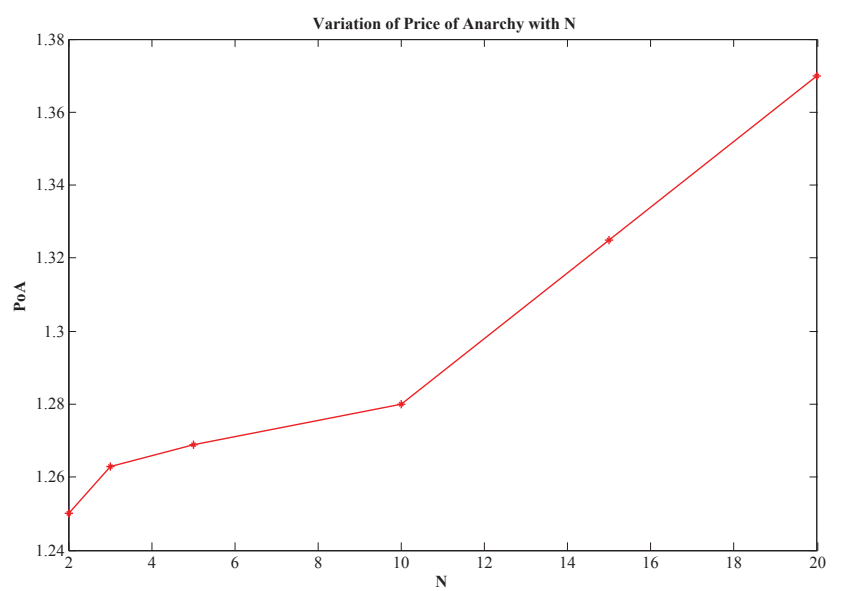

Fig. 4. The variation of $P o A$ with the number of relays $N$.

[3] A. K. Chorppath, T. Alpcan, and H. Boche, "Pricing mechanisms for multi-carrier wireless systems," in in Proc. of IEEE Intl. Dynamic Spectrum Access Networks (DySPAN) Symp., Aachen, Germany, May 2011.

[4] Y. Xi and E. M. Yeh, "Pricing, competition, and routing for selfish and strategic nodes in multi-hop relay networks," in INFOCOM'08, 2008, pp. 1463-1471.

[5] H. Xiao and E. Yeh, "The impact of incomplete information on games in parallel relay networks," IEEE Journal on Selected Areas in Communications, vol. 30, no. 1, pp. 176-187, 2012.

[6] L. Chen, L. Libman, and J. Leneutre, "Conflicts and incentives in wireless cooperative relaying: A distributed market pricing framework," Parallel and Distributed Systems, IEEE Transactions on, vol. 22, no. 5, pp. 758-772, May 2011.

[7] Q. Cao, H. Zhao, and Y. Jing, "Power allocation and pricing in multiuser relay networks using stackelberg and bargaining games," Vehicular Technology, IEEE Transactions on, vol. 61, no. 7, pp. 3177-3190, Sept 2012.

[8] J. Huang, R. Berry, and M. Honig, "Distributed Interference Compensation for Wireless Networks," IEEE Journal on Selected Areas in Communications, vol. 24, no. 5, pp. 1074-1084, May 2006.

[9] R. Johari, S. Mannor, and J. Tsitsiklis, "Efficiency loss in a network resource allocation game: the case of elastic supply," IEEE Transactions on Automatic Control, vol. 50, no. 11, pp. 1712-1724, November 2005

[10] A. K. Chorppath, S. Bhashyam, and R. Sundaresan, "A convex optimization framework for almost budget balanced allocation of a divisible good," IEEE Transactions on Automation Science and Engineering, vol. 8, no. 3, pp. 520-531, July 2011.

[11] T. Başar and G. J. Olsder, Dynamic Noncooperative Game Theory, 2nd ed. Philadelphia, PA: SIAM, 1999.

[12] H. Boche, S. Naik, and T. Alpcan, "Universal pricing mechanism for utility maximization for interference coupled systems," in European Wireless Conference (EW), April 2010, pp. $661-666$. 